\title{
Safety and side effect profile of Pfizer-BioNTech COVID-19 vaccination among healthcare workers: A tertiary hospital experience in Singapore
}

See Ming Lim ${ }^{1}{ }_{M P H}$, Hwang Ching $\underline{\text { Chan }}{ }^{2}{ }_{M P H}$, Amelia Santosa ${ }^{3}{ }_{M B B S}$, Swee Chye Quek ${ }^{4}{ }_{M D}$, Eugene Hern Choon Liu ${ }^{5,6}{ }_{M D}$, Jyoti Somani ${ }^{5,7} M D$

\begin{abstract}
Introduction: Vaccination remains a key strategy to living endemically with COVID-19. The Pfizer-BioNTech COVID-19 vaccine was first granted interim authorisation for use in Singapore in December 2020. With overseas studies published about the safety and side effect profiles of mRNA COVID-19 vaccines focusing mainly on non-Asian populations, we described the side effects of Pfizer-BioNTech COVID-19 vaccination experienced by the healthcare workers (HCWs) in a tertiary hospital in Singapore.

Methods: Data were obtained from the Occupational Health Clinic (OHC) at the National University Hospital in Singapore, which monitored staff for any adverse effects within 30 minutes postvaccination on-site and any adverse effects after that. A cross-sectional study among the vaccinated HCWs was conducted using an online survey, which established basic demographics, histories of allergies or atopic disorders, and adverse events encountered after dose 1 and dose 2 of vaccination.

Results: No anaphylaxis was reported. Most common symptom was giddiness $(32.7 \%)$ experienced by $\mathrm{HCWs}$ within 30 minutes. Adverse events attended post-vaccination by $\mathrm{OHC}$ were generally mild and self-limiting. From the survey, odds of experiencing an adverse event after dose 2 was significantly higher than after the first dose, especially for fever/chills (odds ratio [OR] 22.5). Fever/chills, injection site reactions, headache, aches and pains, and feeling unwell were significantly more common in HCWs below 60 years compared to those $\geq 60$ years. An allergy to food (adjusted OR 2.7) and a history of eczema/sensitive skin (adjusted OR 2.6) were associated with a skin reaction not at injection site.

Conclusion: The side effects experienced after Pfizer-BioNTech COVID-19 vaccines are generally self-limiting and mild, with no anaphylaxis reported.
\end{abstract}

Ann Acad Med Singap 2021;50:703-11

Keywords: COVID-19, infectious diseases, occupational medicine, side effects, vaccination

\section{INTRODUCTION}

The newly emerged coronavirus virus 2019 (COVID-19) disease was declared a Public Health Emergency of International Concern by the World Health Organization (WHO) on 30 January 2020, and subsequently designated a pandemic on 11 March 2020. ${ }^{1}$ Globally, over 209 million cases have been reported, with more than 4.4 million confirmed deaths as of 20 August $2021,{ }^{2}$ with numbers increasing daily.
A series of public health measures including social distancing, wearing of face masks, maintaining good personal hygiene and lockdowns have been introduced to mitigate the spread of the virus. Vaccination, however, remains a key strategy for sustained protection, and living endemically with the virus. ${ }^{3}$

On 11 December 2020, the US Food and Drug Administration issued the first emergency use authorisation for the BNT162b2 COVID-19 vaccine

\footnotetext{
${ }^{1}$ Occupational Health Clinic, National University Hospital, Singapore

${ }^{2}$ Epidemiology Unit, National University Hospital, Singapore

${ }^{3}$ Division of Rheumatology, Department of Medicine, National University Hospital, Singapore

${ }^{4}$ Chairman Medical Board's Office, National University Hospital, Singapore

${ }^{5}$ Yong Loo Lin School of Medicine, National University of Singapore, Singapore

${ }^{6}$ Department of Anaesthesia, National University Hospital, Singapore

${ }^{7}$ Division of Infectious Diseases, Department of Medicine, National University Hospital, Singapore

Correspondence: Dr See Ming Lim, Occupational Health Clinic, National University Hospital, 5 Lower Kent Ridge Road, Main Building, Level 1, Singapore 119074.

Email: see_ming_lim@nuhs.edu.sg
} 


\section{CLINICAL IMPACT}

\section{What is New}

- This study describes the safety and side effect profile of Pfizer-BioNTech COVID-19 vaccination of the healthcare population in Singapore.

- Side effects are generally self-limiting and mild, similar to trials done overseas that comprise mainly non-Asian participants.

\section{Clinical Implications}

- The benefits of vaccination against acquiring COVID-19 infection and its possible complications outweigh the side effects experienced.

- The results from this paper can be used to encourage more to take up COVID-19 vaccination.

developed by Pfizer and BioNTech in individuals aged 16 years and above. ${ }^{4}$ The Pfizer-BioNTech COVID-19 vaccine belongs to a novel category of vaccines called messenger ribonucleic acid (mRNA) vaccines. mRNA vaccines deliver segments of mRNA that encode for a protein, which in the case of the COVID-19 mRNA vaccines, is a code for the spike protein found on the surface of severe acute respiratory syndrome coronavirus 2 (SARS-CoV-2) virus. mRNA is highly degradable, and so these mRNA vaccines have been designed to deliver the mRNA material within liposomes for protection. These liposomes, which contain polyethylene glycol and various other excipients, are highly immunogenic and are thought to be responsible for the strong local and systemic side effects reported in patients. SARS-CoV-2 enters the host airway mucosal cells via the attachment of its spike protein to the host's ACE-II receptor, thus, antibodies to the spike protein are induced by the vaccine, allowing for protection against future infection. ${ }^{5,6}$

There have been concerns among the public and even among health professionals about the safety profile of mRNA vaccines, given that they are new to the market. In the clinical trials of Pfizer-BioNTech COVID-19 vaccines, frequently reported reactions were pain at the injection site, fatigue and headache, which were usually short-term. Systemic reactions were more common and severe after the second dose than after the first dose, and were generally more common in those under the age of 55 compared to older recipients.
The incidence of serious adverse events was low and was similar in both vaccine and placebo groups. ${ }^{7}$

Singapore was the first country in Asia to receive the vaccine on 21 December 2020, and as in many countries, healthcare workers $(\mathrm{HCWs})$ were prioritised to be vaccinated by the Ministry of Health (MOH). However, there remains limited data and literature on the side effects of the Pfizer-BioNTech COVID-19 vaccines that focus on the healthcare population in Asia. Therefore, the objective of this study was to analyse the safety and side effect profile of the Pfizer-BioNTech COVID-19 vaccines among HCWs at the National University Hospital (NUH) in Singapore. The Occupational Health Clinic (OHC) in the tertiary hospital is responsible for administering and monitoring vaccination in newly hired HCWs as well as ensuring timely vaccination updates of all employees. With support from infectious diseases (ID) and allergy specialists, OHC implemented the same institutional processes to monitor $\mathrm{HCWs}$ post-vaccination for the COVID-19 staff vaccination exercise. In addition to 30-minute monitoring post-vaccination on-site, part of the surveillance included a self-reported online survey questionnaire.

\section{METHOD}

$\mathrm{OHC}$ is an in-house clinic within the hospital, which manages work-related health and safety issues of more than 7,600 HCWs to ensure a safe and healthy work environment. OHC, with support from human resources, operations and ID specialists, proactively stepped up its actions at the start of the COVID-19 pandemic to protect staff from contracting COVID-19 at work. Actions included screening and monitoring fitness for work; active redeployment of staff with medical conditions that put them at increased risk of more complicated COVID-19 infection; surveillance of staff health post-exposure; and return-to-work assessment for staff with acute respiratory illnesses.

Working in close collaboration with various medical specialists, nursing, pharmacy and operations teams, OHC officially rolled out the staff COVID-19 vaccination exercise on 11 January 2021, as an important tool to protect the safety and health of staff against COVID-19, in line with organisation and national directives. Some contraindications as listed by $\mathrm{MOH}$ then included immunocompromised conditions, prior anaphylaxis/ severe allergy and pregnancy (see Supplementary Online Materials), which were gradually allowed vaccination as the vaccination exercise continued. 


\section{Monitoring of adverse effects within 30 minutes post- vaccination}

Guidelines from $\mathrm{MOH}$ regarding vaccine preparation, administration, contraindications and side effects were followed, ${ }^{8}$ with a mandatory 30-minute observation period post-vaccination on-site for adverse effects. If any staff developed any symptoms or discomfort during this period, they would be moved to a treatment room for vital sign monitoring and assessment by an $\mathrm{OHC}$ doctor. Based on assessment, unwell staff might be observed for a longer period of time, for example, additional 30 minutes to allow the symptoms to subside. Staff would be transferred to the emergency department (ED) for further evaluation if the symptoms were deemed serious based on initial assessment, for example, severe allergic reactions, or no improvement after extended period of observation.

\section{Monitoring of adverse effects post-vaccination}

$\mathrm{OHC}$ was also responsible for the recording and reporting of any early or late adverse effects and allergic reactions. HCWs were asked to report any symptoms or reactions that occurred later after leaving the vaccination clinic to OHC via calls, emails or walk-in consults. For common side effects such as fever or body ache post-vaccination, appropriate medical advice was given based on $\mathrm{MOH}$ guidelines. ${ }^{8}$ Staff with potential allergic reactions or prolonged/severe side effects were reviewed by $\mathrm{OHC}$ doctors and these problems were reported to $\mathrm{MOH}$. For rashes or side effects that could indicate allergy, OHC and an allergy specialist would evaluate and decide whether a second dose could be given.

\section{Monitoring of adverse effects via online survey}

A cross-sectional study was conducted when HCWs being monitored on-site after the second dose were invited to participate voluntarily in a survey hosted on the form.sg platform, a government-initiated, secure internet database. The questions in part 1 of the survey established basic demographics, histories of allergies (food, medication or vaccines) or atopic disorders (allergic rhinitis, asthma or eczema), and adverse events encountered after the first dose. Those who completed part 1 would be invited to participate in part 2 of the survey 1 week after second dose was administered. Questions in the second part were similarly phrased as those in the first part and included demographics and adverse events encountered after the second dose. Survey responses were collected for a duration of 2 months, between 8 February and 12 April 2021 and analysed using STATA version 13.0 (StataCorp, College Station, US).
Only participants who completed both part 1 and part 2 of the survey were included in the analysis. We used the number of staff who completed 2 doses as the denominator as all were invited to participate post-vaccination (Fig.1). Participants' demographics were descriptive in nature, presented as frequencies and percentages. Differences in responses between the first dose and second dose were compared using McNemar's test of symmetry. Chi-square test was used in hypothesis testing to investigate if there were differences in adverse events reported by different age groups, self-reported histories of allergies (food, medication or vaccines) and atopic disorders (allergic rhinitis, asthma or eczema). We used age strata $<60$ years versus $\geq 60$ years, in line with the national definition of seniors prioritised for receiving COVID-19 vaccinations. Logistic regression was performed with each adverse effect as the dependent variable and a history of allergies or atopic disorders as the independent variable, with crude and adjusted odds ratio (for sex and age as a continuous variable). A $P$ value $<0.05$ was considered statistically significant.

This study was exempted from ethics review by the National Healthcare Group, Domain Specific Institutional Review Board (NHG DSRB reference number 2021/00572).

\section{RESULTS}

\section{Adverse effects attended to at vaccination clinic and at $\mathrm{OHC}$}

The 3 most common symptoms attended to within the 30 -minute observation period at the vaccination clinic

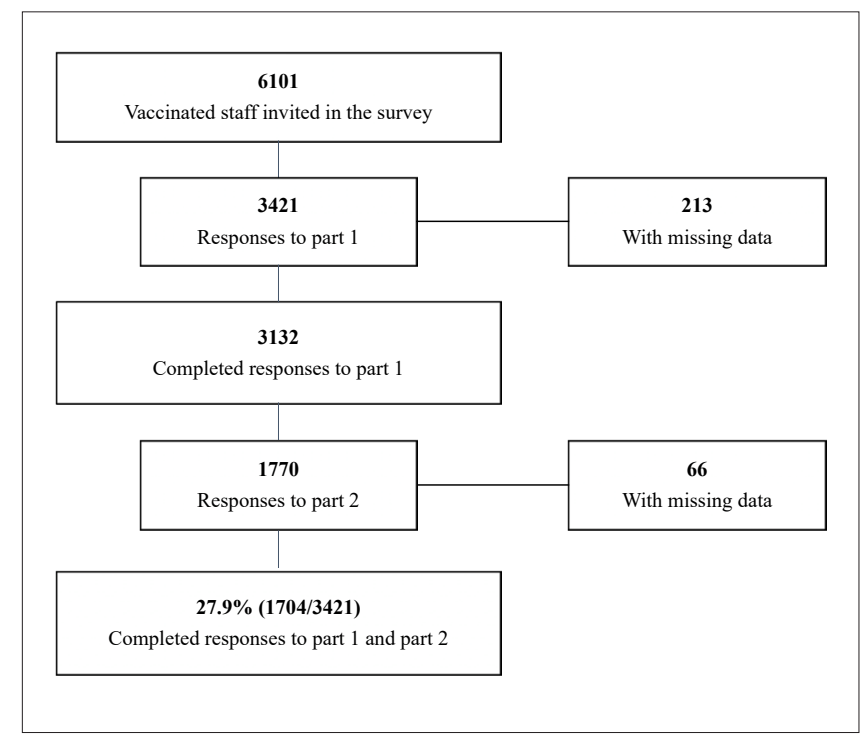

Fig. 1. Flowchart of survey respondents included in the analysis. 
Table 1. Symptoms attended to at the vaccination clinic

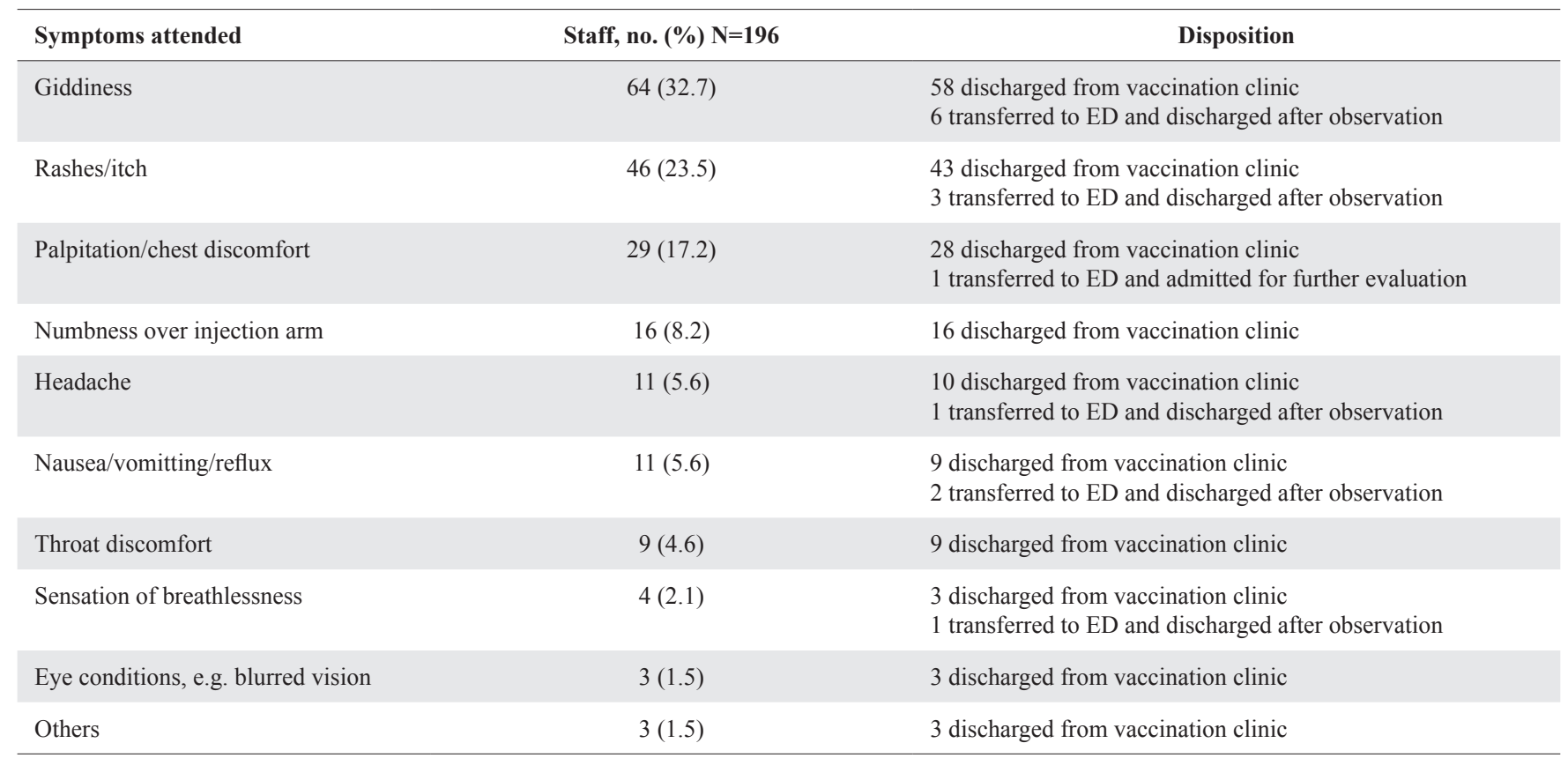

ED: Emergency department

were giddiness $(32.7 \%)$, rashes/itch $(23.5 \%)$ and palpitation/chest discomfort (17.2\%). Most staff who experienced any symptoms improved within 30 minutes of further monitoring. However, of 196 staff requiring such care after vaccination, $16(8.2 \%)$ of them had to be transferred to ED for further evaluation and management. No staff had anaphylaxis (Table 1).

$\mathrm{OHC}$ attended to a total of 329 staff at the end of the staff vaccination exercise. The most common adverse effects attended to were fever/chill (26.1\%), rashes/itch/ eye swelling $(21.3 \%)$, myalgia $(9.4 \%)$, swelling/pain over injection site $(7.6 \%)$, giddiness $(6.1 \%)$ and headache $(6.1 \%)$.

Of all the 525 staff reviewed at $\mathrm{OHC}$ and vaccination clinic, 28 staff were advised to withhold their second doses due to angioedema (35.7\%), urticaria $(35.7 \%)$, pregnancy $(25.0 \%)$ and retinal clots/hypertension (3.6\%), while 10 other staff were advised against future doses due to angioedema $(50.0 \%)$ and urticaria $(50.0 \%)$ after second dose. The pregnant staff were subsequently allowed to complete dose 2 after $\mathrm{MOH}$ revised its guidance. ${ }^{9}$

\section{Adverse reactions reported from survey}

A total of 3,421 out of 6,101 vaccinated staff responded to part 1 of the survey, of which 1,704 completed part 2 as well, giving a response rate of $27.9 \%$ (Fig. 1). Among the respondents to the survey, those $18-50$ years of age accounted for the large majority (87.4\%), while those 51-80 years old made up the remaining $12.6 \%$. Respondents' median age was 35 years old, with a range of 18-76 years. The ethnicity of those who responded to the survey also corresponded proportionally with the overall makeup of the healthcare workers in our institution, with Chinese making up the majority ethnicity at $55.6 \%$. There were also more female respondents $(78.6 \%)$ than male, which relates proportionally to the overall gender ratio in our hospital settings (Table 2). At the time of vaccination, no HCWs in this study were documented to have had COVID-19.

A majority of respondents reported localised injection site reactions, with more experiencing such reactions after dose 2 (70.1\%) compared to dose 1 (57.2\%). Feeling unwell in general, having aches and pains, headache, and fever or chills were also commonly reported symptoms (Table 3). Although anaphylaxis was included in the survey as a symptom, none of the respondents experienced this as an adverse event. The odds of experiencing an adverse event after the second dose was significantly higher than after the first dose. Although all adverse events were reported significantly more likely after the second dose, having fever/chills was the most striking, with an odds ratio of 22.5 on the second dose, compared to the first.

Among the adverse events included in the survey, having fever or chills, injection site reactions, headache, 
Table 2. Demographics of survey respondents

\begin{tabular}{|c|c|c|}
\hline & $\begin{array}{c}\text { Numbers responded } \\
\qquad(\mathrm{N}=1704)\end{array}$ & $\begin{array}{c}\text { Percentage of } \\
\text { respondents (\%) }\end{array}$ \\
\hline \multicolumn{3}{|c|}{ Age group (years) } \\
\hline $18-30$ & 494 & 29.0 \\
\hline $31-40$ & 649 & 38.1 \\
\hline $41-50$ & 346 & 20.3 \\
\hline $51-60$ & 160 & 9.4 \\
\hline $61-70$ & 50 & 2.9 \\
\hline $71-80$ & 5 & 0.3 \\
\hline \multicolumn{3}{|l|}{ Sex } \\
\hline Female & 1340 & 78.6 \\
\hline Male & 364 & 21.4 \\
\hline \multicolumn{3}{|l|}{ Ethnicity } \\
\hline Chinese & 948 & 55.6 \\
\hline Malay & 198 & 11.6 \\
\hline Indian & 174 & 10.2 \\
\hline Eurasian & 9 & 0.5 \\
\hline Others & 375 & 22.0 \\
\hline \multicolumn{3}{|l|}{ Job groups } \\
\hline Administration & 194 & 11.4 \\
\hline Allied health & 323 & 19.0 \\
\hline Ancillary & 151 & 8.9 \\
\hline Medical/Dental & 203 & 11.9 \\
\hline Nursing & 693 & 40.7 \\
\hline Service partners & 50 & 2.9 \\
\hline MOHH doctors & 16 & 0.9 \\
\hline Others & 74 & 4.3 \\
\hline
\end{tabular}

MOHH: Ministry of Health Holdings

generalised aches and pains and feeling unwell were significantly more common in the younger age group. The survey showed $47.2 \%$ of respondents below 60 years reported fever or chills after dose 1 and/or dose 2 , compared to $25.0 \%$ in those aged 60 and above $(P<0.01)$. A majority $(78.5 \%)$ of those in the age group below 60 years reported an injection site reaction, while the prevalence among those 60 years and above was comparatively lower at $47.1 \%(P<0.01)$. Feeling unwell, aches and pains, and headache were also symptoms more significantly reported in those below 60 years, compared to those 60 years and above (Fig. 2).
Among survey respondents who self-reported an allergy (food, medication or vaccines) or atopic disorders (allergic rhinitis/sensitive nose, asthma, or eczema/sensitive skin), an allergy to food, and a history of eczema/sensitive skin were the only 2 conditions associated with a higher odds ratio of skin reaction not at the injection site (rash, hives, urticaria or itch). After adjusting for age and gender, the odds ratio of respondents with an allergy to a food and reporting a skin reaction post-vaccination was 2.7 that of those without any food allergy, while the odds ratio of respondents with eczema/sensitive skin was 2.6 that of respondents who reported no eczema/sensitive skin (Table 4). Both were statistically significant.

\section{DISCUSSION}

This study aimed to analyse the safety and side effect profile of the Pfizer-BioNTech COVID-19 vaccines among HCWs at NUH in Singapore. Based on the survey results, localised injection site reactions (rash, redness, swelling and pain) were the most common, followed by systemic reactions such as feeling unwell in general (fatigue, tiredness and weakness) and aches and pains (joint pain, muscle pain and body ache). Reactions were generally self-limiting in nature and were reported more often after dose 2 than dose 1 , and in the younger age group compared to the older age group. The findings were consistent with those observed in studies conducted overseas and reports of suspected adverse events in Singapore as well. ${ }^{7,10,11}$

No anaphylaxis of HCWs vaccinated in the hospital was reported as of 24 August 2021. As of 31 July 2021, the incidence of anaphylaxis reported in Singapore with the mRNA vaccines was about 0.86 per 100,000 doses administered, similar to those reported overseas of around 0.5 to 2 per $100,000 .^{11,12}$ We reported no cases of myocarditis/pericarditis.

Since mRNA vaccination started in Singapore, $\mathrm{MOH}$ had taken a more conservative stance with more contraindications and precautions for who could be vaccinated initially (see Supplementary Online Materials). With the emergence of international data from real-world vaccination roll-outs, contraindications were gradually relaxed after no major safety concerns were noted. For example, persons with history of allergic reactions (excluding to vaccines) not amounting to an anaphylaxis were allowed to be vaccinated after 12 March 2021, while those with history of anaphylaxis were excluded until 5 June $2021,{ }^{13}$ after no evidence to suggest that a prior history of anaphylaxis would predict the development of anaphylaxis to the mRNA COVID-19 vaccines. 


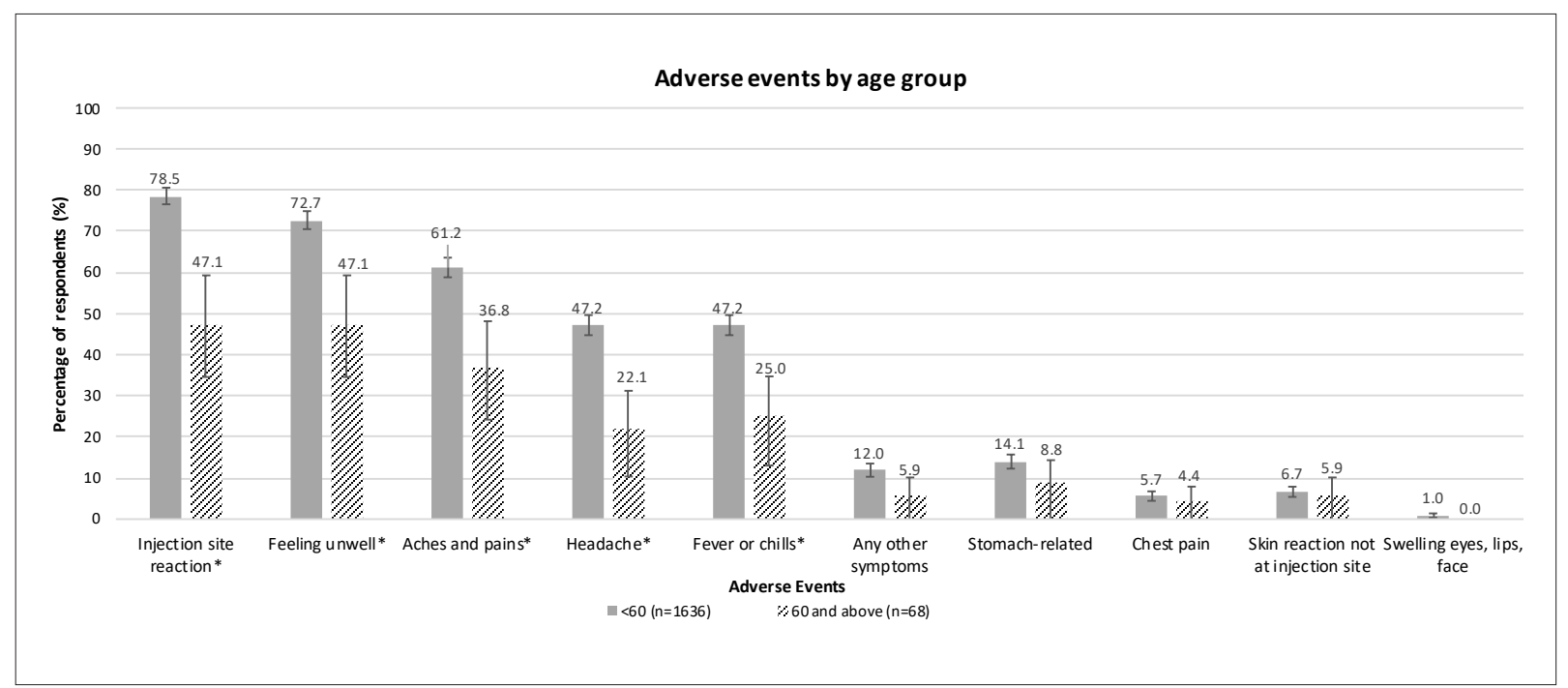

Fig. 2. Adverse events reported after dose 1 and/or dose 2, by age group.

* Significantly different proportions between respondents aged $<60$, and those aged 60 and above, who reported the specified adverse effect (chi-squared test for statistical significance, $P<0.01$ )

I bars represent $95 \%$ confidence intervals, and number labels indicate the percentage of participants who reported the stated adverse event.

Table 3. Comparison of adverse reactions reported after dose 1 and dose 2

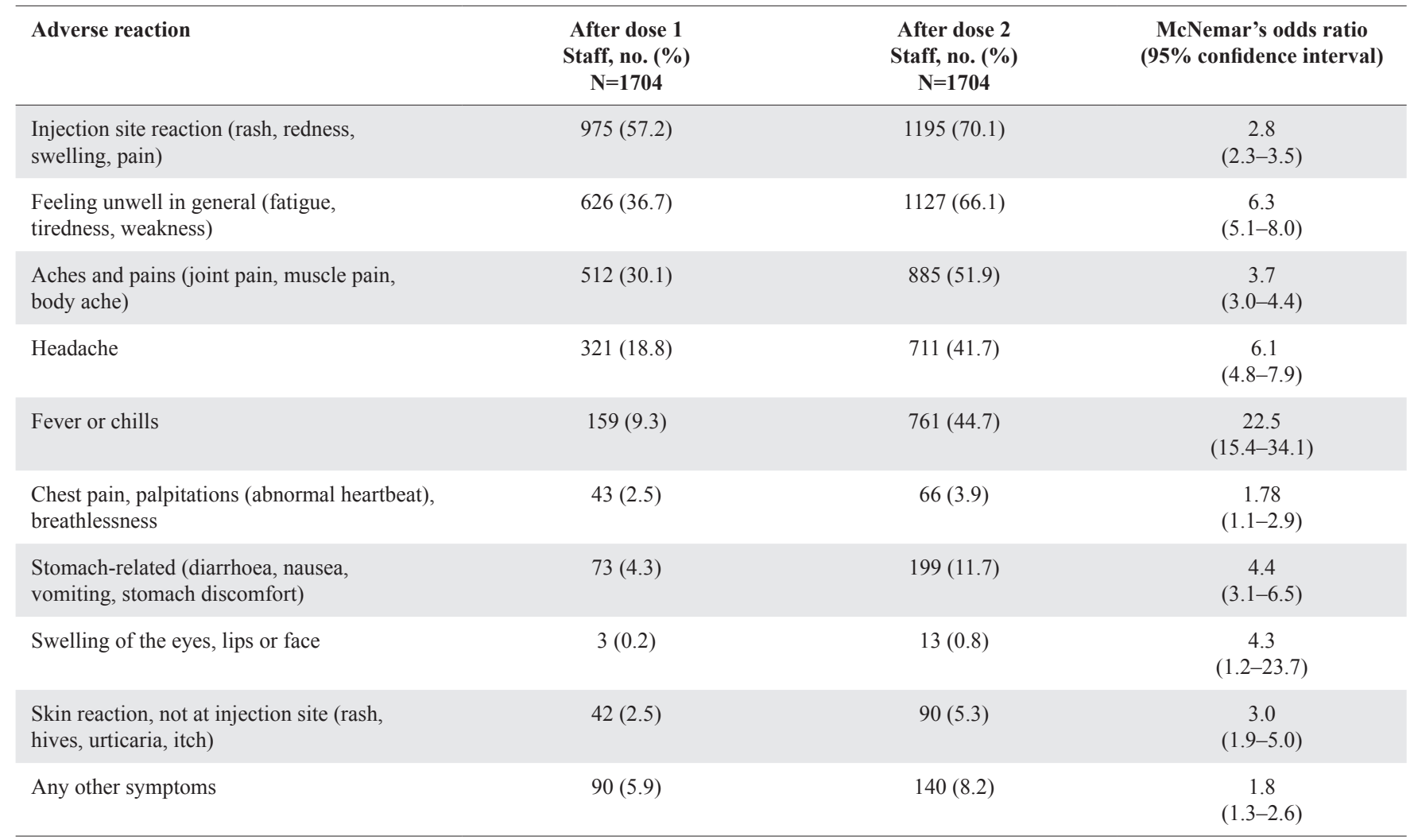

A self-reported history of allergy to food and eczema/ sensitive skin were associated with a higher odds ratio of patient-reported skin reaction not at injection site (rash, hives, urticaria or itch), after adjusting for age and gender. As the nature of the previous allergies and the current reactions were reported by the staff themselves, it is difficult to comment whether this association is truly mediated by an allergic predisposition, 
Table 4. Effect of allergy/atopic disorders on skin reaction and face swelling post-vaccination

\begin{tabular}{|c|c|c|c|c|c|c|}
\hline & \multicolumn{3}{|c|}{$\begin{array}{l}\text { Skin reaction not at injection site } \\
\text { (rash, hives, urticaria, itch) }\end{array}$} & \multicolumn{3}{|c|}{ Swelling of eyes, lips, face } \\
\hline & $\begin{array}{l}\text { Proportion of } \\
\text { respondents } \\
(\%)\end{array}$ & $\begin{array}{l}\text { Unadjusted } \\
\text { odds ratio } \\
\text { (95\% CI) }\end{array}$ & $\begin{array}{c}\text { Adjusted odds } \\
\text { ratio } \\
(95 \% \mathrm{CI})\end{array}$ & $\begin{array}{c}\text { Proportion of } \\
\text { respondents } \\
(\%)\end{array}$ & $\begin{array}{l}\text { Unadjusted } \\
\text { odds ratio } \\
(95 \% \text { CI) }\end{array}$ & $\begin{array}{c}\text { Adjusted } \\
\text { odds ratio }^{\mathrm{a}} \\
(95 \% \mathrm{CI})\end{array}$ \\
\hline \multicolumn{7}{|l|}{ Allergies } \\
\hline Allergy to food $(\mathrm{n}=85)$ & 15.3 & $2.7(1.5-5.1)^{\mathbf{b}}$ & $2.7(1.4-5.0)^{\mathrm{b}}$ & 0 & - & - \\
\hline No allergy to food $(\mathrm{n}=1619)$ & 6.2 & & & 1.0 & & \\
\hline Allergy to medication $(\mathrm{n}=185)$ & 5.4 & $0.8(0.4-1.5)$ & $0.8(0.4-1.5)$ & 1.1 & $0.8(0.4-1.5)$ & $0.8(0.4-1.5)$ \\
\hline No allergy to medication $(\mathrm{n}=1519)$ & 6.8 & & & 0.9 & & \\
\hline Allergy to vaccines $(\mathrm{n}=9)$ & 11.1 & $1.8(0.2-14.1)$ & $1.5(0.2-12.4)$ & 0 & - & - \\
\hline No allergy to vaccines ( $\mathrm{n}=1695$ ) & 6.7 & & & 0.9 & & \\
\hline \multicolumn{7}{|l|}{ Atopic disorders } \\
\hline Allergic rhinitis/sensitive nose $(n=322)$ & 9.0 & $1.5(0.9-2.3)$ & $1.4(0.9-2.3)$ & 1.2 & $1.4(0.5-4.5)$ & $1.4(0.4-4.4)$ \\
\hline No allergic rhinitis/sensitive nose $(\mathrm{n}=1382)$ & 6.2 & & & 0.9 & & \\
\hline Asthma $(\mathrm{n}=183)$ & 9.8 & $1.6(0.9-2.8)$ & $1.6(0.9-2.8)$ & 1.1 & $1.2(0.3-5.3)$ & $1.2(0.3-5.4)$ \\
\hline No asthma $(\mathrm{n}=1521)$ & 6.3 & & & 0.9 & & \\
\hline Eczema/sensitive skin $(n=287)$ & 12.9 & $2.6(1.7-3.9)^{\mathbf{b}}$ & $2.5(1.7-3.9)^{\mathbf{b}}$ & 0.7 & $0.7(0.2-3.1)$ & $0.7(0.2-3.1)$ \\
\hline No eczema/sensitive skin $(n=1417)$ & 5.4 & & & 1.0 & & \\
\hline
\end{tabular}

a Adjusted for age as a continuous variable and sex

b Statistically significant odds ratio $(P<0.01)$

or whether patients may suffer from other pre-existing conditions such as chronic urticaria or anxiety, which may confound the interpretation of the reactions. ${ }^{14,15}$

Overall, the approach we took to monitor for adverse events for the staff vaccination exercise was safe and in line with $\mathrm{MOH}$ guidelines, particularly considering that it was rolled out in the context of an Emergency Use Approval as a very new type of vaccine with little prior clinical experience especially among Asian population, with only $4.3 \%$ included in phase $2 / 3$ trials. ${ }^{7}$ On-site monitoring for adverse effects within 30 minutes of vaccination is sufficient for diagnosis and treatment of early onset allergic reactions or anaphylaxis. The observation of frequent giddiness, palpitation/chest discomfort and headache post-vaccination also led to staff being reminded to adhere to their chronic medications and hydrate appropriately early on the day of vaccination.

After leaving the vaccination clinic, HCWs with any adverse effects were encouraged to report to $\mathrm{OHC}$ via phone call, email or walk-in consults. Those with common adverse effects such as fever, myalgia or pain over the injection sites were given post-vaccination management advice as per $\mathrm{MOH}$ guidelines. ${ }^{8}$ Staff with potential allergic reactions or prolonged/severe side effects were reviewed as a walk-in consult at OHC. HCWs were encouraged to take photos of any visible reaction (such as rashes) to show them during these consultations. All staff who presented in this manner were reviewed by the same $\mathrm{OHC}$ specialist in the clinic, and also evaluated by a single allergy specialist. This would allow objective reporting and reduce observation bias between cases.

By the end of the vaccination exercise on 26 March 2021 , nearly $80 \%(6,101$ out of 7,671$)$ of staff had completed both doses of the vaccine and subsequent vaccination of staff was by appointment only. When Singapore experienced a surge of community cases end April 2021, walk-in slots were made available for staff, which subsequently coincided with MOH's loosening of the list of contraindications to vaccination. With this, staff vaccination rates reached $94 \%$ as of 24 August 2021. While these conditions were gradually relaxed, it likely impacted vaccine acceptance, particularly among pregnant and breastfeeding $\mathrm{HCWs}$ and those trying to conceive, as $86(25.3 \%)$ out of 340 staff who remained unvaccinated gave pregnancy-related reasons and breastfeeding for why they were not vaccinated. 
Unvaccinated staff had to be managed carefully. At the start of the pandemic, staff with chronic medical conditions that put them at increased risk of more complicated COVID-19 infection were already redeployed to non-COVID-19 patient areas. ${ }^{16}$ Strict compliance to personal protective equipment (PPE) and hand hygiene was enforced regularly. Similarly, unvaccinated staff were placed in lower risk areas with an emphasis on PPE compliance, while efforts to get more staff vaccinated were ongoing.

With evidence that immunity may decline over time, the Expert Committee on COVID-19 Vaccination in Singapore had recommended an additional mRNA COVID-19 vaccine dose for the immunocompromised and seniors. ${ }^{17}$ However, in the US, HCWs who were vaccinated earliest in the vaccination roll-out would be eligible for booster dose as well. ${ }^{18}$ Further research may be required to determine the optimal timing of the booster for HCWs that will ensure maximal protection exceeds the risk of untoward side effects. ${ }^{19}$

This study has a few limitations. Participation rate with valid responses was $27.9 \%$ for this self-reporting survey. It is possible that those who did not participate have milder adverse reactions compared to those who participated. Also as the first part was administered 3 weeks after the first dose, and the second part a week after dose 2, the time between experiencing the effects and the collection of participants' responses to the survey could lead to recall error. We had tried to minimise this by constructing survey questions that were welldefined and easily understood, and piloted this among a convenient sampling of healthcare workers. Even then, we identified this as a limitation, and took into consideration other studies in conjunction with our findings, before drawing conclusions from our survey results.

\section{CONCLUSION}

In conclusion, the side effects experienced after PfizerBioNTech COVID-19 vaccines are generally selflimiting and non-severe, with no anaphylaxis reported among the HCWs in NUH, Singapore. The benefits of vaccination against acquiring COVID-19 infection and its possible complications far outweigh the self-limiting side effects experienced. However, further studies to determine the longer-term adverse effects of this relatively new vaccine would enable individuals to make a more confident informed choice, and lend more evidence to guide public health practitioners on vaccination policy for the wider population.

\section{Acknowledgements}

We thank our fellow healthcare colleagues from nursing, pharmacy, operations centre, administration and the Occupational Health Clinic who operated the staff vaccination clinic in National University Hospital. We also thank our senior management for their support.

\section{REFERENCES}

1. World Health Organization. Rolling updates on coronavirus disease (COVID-19), 31 July 2020. Available at: https:/www.who.int/ emergencies/diseases/novel-coronavirus-2019/events-as-they-happen. Accessed on 22 August 2021.

2. World Health Organization. Coronavirus disease (COVID-19) pandemic. Available at: https://www.who.int/emergencies/diseases/ novel-coronavirus-2019?gclid=CjwKCAjwyIKJBhBPEiwAu7zll4H KIeSbTVhCS3oiq33vDRMtwYyLXUqZuhy1y7zl9wCea6W16tM56 BoCWGkQAvD_BwE. Accessed on 22 August 2021.

3. World Health Organization. COVID-19 vaccines. Available at: https://www.who.int/emergencies/diseases/novel-coronavirus-2019/ covid-19-vaccines. Accessed on 22 August 2021.

4. US Food and Drug Administration. Pfizer-BioNTech COVID-19 Vaccine, 23 August 2021. Available at: https://www.fda.gov/emergencypreparedness-and-response/coronavirus-disease-2019-covid-19/pfizerbiontech-covid-19-vaccine. Accessed on 22 August 2021.

5. Centers for Disease Control and Prevention. mRNA vaccines, 4 March 2021. Available at: https://www.cdc.gov/coronavirus/2019ncov/vaccines/different-vaccines/mRNA.html. Accessed on 22 August 2021.

6. Banerji A, Wickner PG, Saff R, et al. mRNA Vaccines to Prevent COVID-19 Disease and Reported Allergic Reactions: Current Evidence and Suggested Approach. J Allergy Clin Immunol Pract 2021;9:1423-37.

7. Polack FP, Thomas SJ, Kitchin N, et al. Safety and Efficacy of the BNT162b2 mRNA Covid-19 Vaccine. N Engl J Med 2020;383:2603-15.

8. Ministry of Health, Singapore. COVID-19 vaccination. Available at: https://www.moh.gov.sg/covid-19/vaccination. Accessed on 22 August 2021

9. Ministry of Health, Singapore. Expert Committee on COVID-19 Vaccination expands medical eligibility for mRNA vaccines, 31 May 2021. Available at: https://www.moh.gov.sg/news-highlights/ details/expert-committee-on-covid-19-vaccination-expands-medicaleligibility-for-mrna-vaccines. Accessed on 1 September 2021.

10. Kadali RAK, Janagama R, Peruru S, et al. Side effects of BNT162b2 mRNA COVID-19 vaccine: A randomized, crosssectional study with detailed self-reported symptoms from healthcare workers. Int J Infect Dis 2021;106:376-81

11. Health Sciences Authority, Singapore. HSA's Safety Update \#4 COVID-19 vaccines (30 December 2020 - 31 July 2021), 31 July 2021. Available at: https://www.hsa.gov.sg/docs/default-source/hprg-vcb/ safety-update-on-covid19-vaccines/hsa-safety-update-no-4-on-covid19-vaccines-(31-july-2021).pdf. Accessed on 1 September 2021.

12. Shavit R, Maoz-Segal R, Iancovici-Kidon M, et al. Prevalence of Allergic Reactions After Pfizer-BioNTech COVID-19 Vaccination Among Adults With High Allergy Risk. JAMA Netw Open 2021;4:e2122255.

13. Ministry of Health, Singapore. Safety of mRNA vaccines in persons with anaphylaxis. Available at:https:/www.moh.gov.sg/news-highlights/ details/safety-of-mrna-vaccines-in-persons-with-anaphylaxis. Accessed on 1 September 2021. 
14. Langan SM, Irvine AD, Weidinger S. Atopic dermatitis. Lancet 2020;396:345-60.

15. Sicherer SH, Sampson HA. Food allergy: Epidemiology, pathogenesis, diagnosis, and treatment. J Allergy Clin Immunol 2014;133:291-307.

16. Hwang J, Yong E, Cheong K, et al. Responding to the COVID-19 pandemic: The role of occupational health services in a tertiary hospital in Singapore. J Occup Health 2020;62:e12172.

17. Ministry of Health, Singapore. Expert Committee on COVID-19 Vaccination recommends an additional dose of mRNA COVID-19 vaccine for immunocompromised and seniors, 3 September 2021. Available at: https://www.moh.gov.sg/news-highlights/details/expertcommittee-on-covid-19-vaccination-recommends-an-additional- dose-of-mrna-covid-19-vaccine-for-immunocompromised-andseniors_3Sep2021. Accessed on 5 September 2021.

18. US Department of Health and Human Services. Joint Statement from HHS Public Health and Medical Experts on COVID-19 Booster Shots, 18 August 2021. Available at: https://www.hhs. gov/about/news/2021/08/18/joint-statement-hhs-public-healthand-medical-experts-covid-19-booster-shots.html. Accessed on 5 September 2021.

19. Centers for Disease Control and Prevention. Overview of data to inform recommendations for booster doses of COVID-19 vaccines, 23 June 2021. Available at: https://www.cdc.gov/vaccines/acip/meetings/ downloads/slides-2021-06/06-COVID-Oliver-508.pdf. Accessed on 4 September 2021. 